\title{
Lattice QCD simulations
}

\author{
Karl Jansen*广 \\ NIC, Platanenallee 6, 15738 Zeuthen, Germany \\ E-mail: karl.jansen@desy.de
}

In this contribution we show the recent progress that has been achieved in lattice QCD simulations. We then compare results, as obtained from the European twisted mass collaboration as an example, for the light meson sector for the case when only a mass degenerate light quark doublet $\left(N_{f}=2\right)$ is used and the situation where a dynamical strange and charm quark degree of freedom $\left(N_{f}=2+1+1\right)$ is included.

13th International Workshop on Advanced Computing and Analysis Techniques in Physics Research, ACAT2010

February 22-27, 2010

Jaipur, India

*Speaker.

${ }^{\dagger}$ A footnote may follow. 
In nature, we observe three generations of neutrinos as demonstrated by LEP. Correspondingly, there should exist 3 generations of quarks, ordered in quark pairs as (up,down), (strange, charm), (bottom,top). The masses of these quarks show a particular hierarchy with essentially mass-degenerate up and down quarks and basically exponentially increasing mass values from the strange to the top quark.

We strongly believe nowadays that the quarks are the constituents of all hadrons with the gluons being the interaction particles that -via the strong interaction- "glue" the quarks together to form the bound hadron states which are the particles observed in experiments. The mechanism of forming the bound states is theoretically described by quantum chromodynamics. The postulation of QCD is that at very short distances the quarks behave as almost free particles that interact only very weakly, a phenomenon we call asymptotic freedom. At large distances, at the order of $1 \mathrm{fm}$, the quarks interact extremely strong and in fact so strong that they will never be seen as final, observable states but rather form the observed hadron bound spectrum. The latter phenomenon is called confinement of quarks.

Since the interaction between quarks become so strong at large distances, analytical methods such as perturbation theory fail to analyze QCD. A method to nevertheless tackle the problem is to formulate QCD on a 4-dimensional, euclidean space-time grid. This setup first of all allows for a rigorous definition of QCD and leads to fundamental theoretical and conceptual investigations. On the other hand, the lattice approach enables theorists to perform large scale numerical simulations. In this contribution, we will describe one approach to "lattice QCD", the twisted mass formulation.

In the past, lattice physicists had to work with a number of limitations when performing numerical simulations. These simulations are extremely expensive, reaching the need for Petaflop computing and even beyond, a regime of computing power we just reach today. Therefore, for a long time the quarks were treated as infinitely heavy, indeed a crude approximation given that the up and down quarks have masses of only $\mathrm{O}(\mathrm{MeV})$. In a next step, only the lightest quark doublet, the up and down quarks, were taken into consideration, although their mass values as used in the simulation had been unphysically large.

Nowadays, besides the up and down quarks, also the strange quark is included in the simulations. In addition, these simulations are performed in almost physical conditions, having the quark masses close to their physical values, large lattices with about $3 \mathrm{fm}$ linear extent and small values of the lattice spacing such that a controlled continuum limit can be performed. The situation of the change of the simulation landscape is illustrated in fig. 1(a). In the figure, the blue dot indicates the physical point. The black cross represents a state of the art simulation in the year 2001. As can be seen in the graph, most of the simulations now go well beyond what could be reached in 2001 demonstrating clearly the progress in performing realistic simulations.

The drastic change in the situation is due to three main developments: $i$ ) algorithmic breakthroughs; ii) machine development; the computing power of the present BG/P systems is even outperforming Moore's law, iii) conceptual developments, such as the use of improved actions which reduce lattice artefacts and the development of non-perturbative renormalization.

As a physical example of results we can achieve presently, we show in fig. 1(b) the continuum extrapolated strange baryon spectrum as obtained by the BMW collaboration [28] and the European Twisted Mass Collaboration (ETMC) [1] of which the author is a member. ETMC comprises 16 institutions in Europe, i.e. Cyprus (Univ. of Cyprus), France (Univ. of Paris Sud and LPSC Greno- 


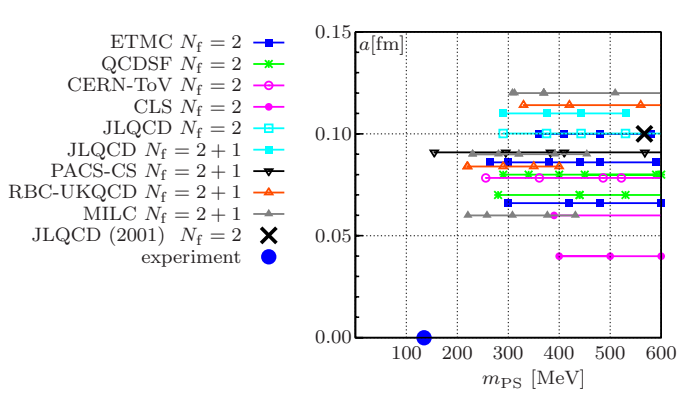

(a)

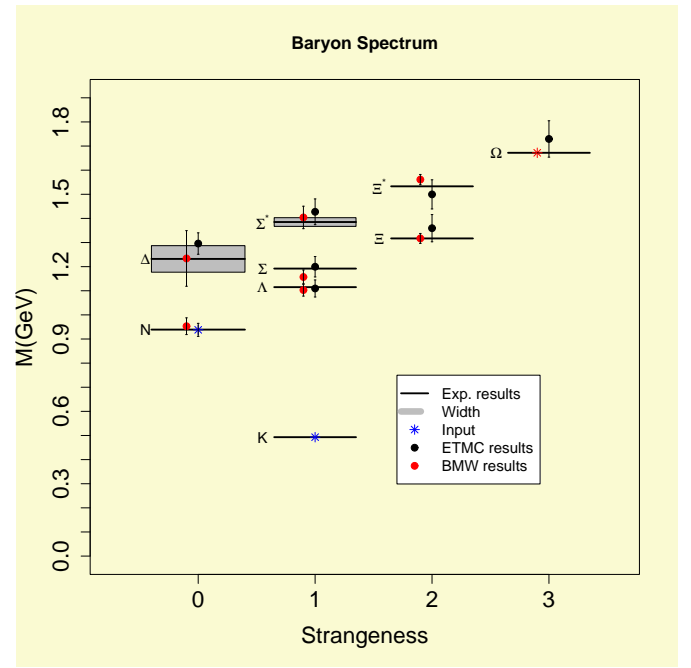

(b)

Figure 1: (a) The values of the lattice spacing $a$ and pseudo scalar masses $m_{\mathrm{PS}}$ as employed presently in typical QCD simulations by various collaborations as (incompletely) listed in the legend. The blue dot indicates the physical point where in the continuum the pseudo scalar meson assumes its experimentally measured value. The black cross represents a state of the art simulation by the JLQCD collaboration in 2001. (b) The continuum strange baryon spectrum from the ETM collaboration [1] using $N_{f}=2$ and the BMW collaborations [28] using $N_{f}=2+1$ flavours of quarks.

ble), Germany (Humboldt Univ. zu Berlin, Univ. of Münster, DESY in Hamburg and Zeuthen), Great Britain (Univ. of Glasgow and Univ. of Liverpool), Italy (Univ. of Rome I, II and III, ECT* Trento), Netherlands (Univ. of Groningen), Poland, (Univ. of Poznan), Spain (Univ. of Valencia), Switzerland (Univ. of Bern).

The baryon spectrum calculation has been considered a benchmark study for lattice QCD for a long time. It is therefore very reassuring that finally this important result can be obtained precisely from ab-initio and non-perturbative lattice simulations.

\section{Twisted mass fermions}

Twisted mass fermions [3, 4] belong to the class of Wilson fermions [2]. In this approach the lattice artefacts in physical observables appear only quadratic in the lattice spacing when the theory is tuned to the so-called maximal twist situation, see below. This is in contrast to a standard Wilson action, where these lattice spacing effects are linear. The main advantage of the twisted mass formulation of lattice QCD is then that these kind of fermions provide an improved, i.e. $\mathscr{O}\left(a^{2}\right)$, continuum limit scaling of physical observables. The twisted mass formulation of Lattice QCD [3,4] is being studied extensively with $N_{f}=2$ dynamical flavours, i.e. including only the lightest up and down quark doublet [8] as well by including a dynamical strange and charm quark degree of freedom $\left(N_{f}=2+1+1\right)[19,26,27]$ by the European Twisted Mass collaboration.

The fermionic action for two flavours of twisted, mass degenerate quarks in the so called twisted basis $[3,9]$ reads

$$
S_{\mathrm{tm}}=a^{4} \sum_{x}\left\{\bar{\chi}(x)\left[D[U]+m_{0}+i \mu_{q} \gamma_{5} \tau^{3}\right] \chi(x)\right\}
$$


where $m_{0}$ is the untwisted bare quark mass, $\mu_{q}$ is the bare twisted quark mass, $\tau^{3}$ is the third Pauli matrix acting in flavour space and

$$
D[U]=\frac{1}{2}\left[\gamma_{\mu}\left(\nabla_{\mu}+\nabla_{\mu}^{*}\right)-a \nabla_{\mu}^{*} \nabla_{\mu}\right]
$$

is the mass-less Wilson-Dirac operator. $\nabla_{\mu}$ and $\nabla_{\mu}^{*}$ are the forward and backward gauge covariant difference operators, respectively. Twisted mass fermions are said to be at maximal twist if the bare untwisted quark mass $m_{0}$ is tuned to its critical value $m_{\text {crit }}$, the situation we shall be interested in. For convenience we define the hopping parameter $\kappa=1 /\left(8+2 a m_{0}\right)$.

Maximally twisted mass fermions provide important advantages: the spectrum of $Q^{\dagger} Q$ with $Q=\gamma_{5}\left(D[U]+m_{0}+i \mu_{q} \gamma_{5}\right)$ is bounded from below, which was the original reason to consider twisted mass fermions [3]. At maximal twist, the twisted quark mass $\mu_{q}$ is related directly to the physical quark mass and renormalises multiplicatively only. Many mixings under renormalisation are expected to be simplified $[9,4]$. And, physical observables are automatically $\mathscr{O}(a)$ improved. Another feature of maximally twisted mass fermions is that the pseudo scalar decay constant $f_{\mathrm{PS}}$ does not need any renormalisation which allows for a very precise determination of this quantity.

The main drawback of maximally twisted mass fermions is that both parity and flavour symmetry are broken explicitly at non-zero values of the lattice spacing. However, it turns out that this is most probably only relevant for the mass of the neutral pseudo scalar meson (and kinematically related quantities) [10].

\section{Results for two flavours of mass-degenerate quarks}

Since in the maximal twist situation the theory is $\mathscr{O}(a)$-improved, leading lattice artefacts are expected to be of order $a^{2}$. This can be checked by extrapolating a physical quantity in units of the force parameter [11] $r_{0}^{\chi}$ extrapolated to the chiral limit at fixed physical situation to the continuum limit. We show two such examples in figure 2. In the left panel we show $r_{0}^{\chi} f_{\mathrm{PS}}$ as a function of $\left(a / r_{0}^{\chi}\right)^{2}$ at fixed value of $r_{0}^{\chi} m_{\mathrm{PS}}$. In order to match the values of $r_{0}^{\chi} m_{\mathrm{PS}}$ at each value of $r_{0}^{\chi} / a$ and to fix the volume to $r_{0}^{\chi} \cdot L=5$ we had to perform short inter- or extra-polations. The straight lines are linear fits in $\left(a / r_{0}^{\chi}\right)^{2}$ to the corresponding data, with the data at the largest value of the lattice spacing not being included in the fit. It is clearly visible that the lattice artefacts appear to scale linearly in $a^{2}$ and that their overall size is small.

In the right panel of figure 2 we show the scaling of $r_{0}^{\chi} m_{\mathrm{PS}}$ as a function of $\left(a / r_{0}^{\chi}\right)^{2}$ at fixed values of the renormalised quark mass $r_{0}^{\chi} \mu_{R}$, again at fixed, finite volume. We conclude that also the charged pseudo scalar mass has only small lattice artefacts.

The dependence of $m_{\mathrm{PS}}$ and $f_{\mathrm{PS}}$ on the renormalised quark mass and volume can be described by chiral perturbation theory $(\chi \mathrm{PT})[13,14]$. The residual lattice artefacts of order $a^{2}$ can also be included in the analysis. The corresponding formulae can be found in Ref. [15, 8]. We fit these formulae to our data in order to extract the parameters of the $N_{f}=2$ chiral Lagrangian, i.e. the low energy constants and some derived quantities. Moreover, we can use these fits to calibrate our lattices by determining the value of the renormalised quark mass $r_{0}^{\chi} \mu_{R}$ where the ratio $m_{\mathrm{PS}} / f_{\mathrm{PS}}$ assumes its physical value (i.e. $m_{\pi} / f_{\pi}$ ) and set $f_{\mathrm{PS}}=f_{\pi}=130.7 \mathrm{MeV}$ there, as done in ref. [5]. Hence, $f_{\pi}$ is used in this paper to set the scale. 


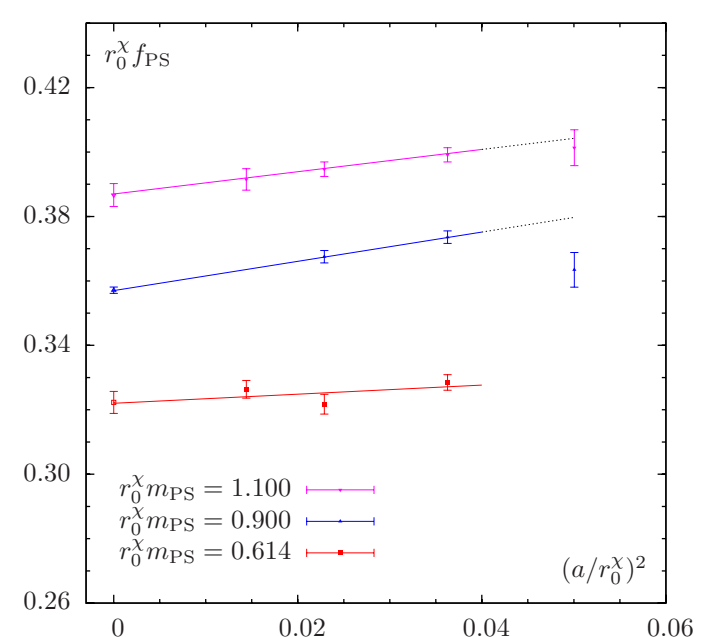

(a)

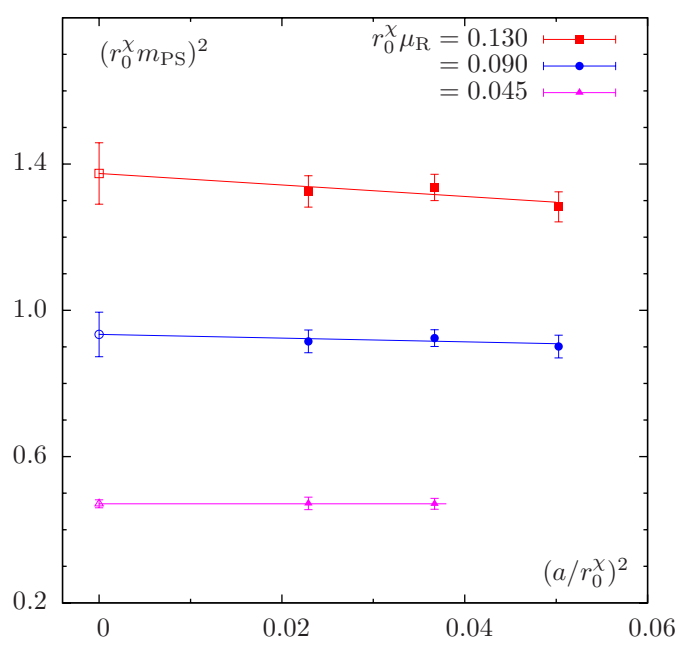

(b)

Figure 2: Scaling in finite, fixed volume for $r_{0}^{\chi} f_{\mathrm{PS}}$ at fixed values of $r_{0}^{\chi} m_{\mathrm{PS}}$ (a) and for $\left(r_{0}^{\chi} m_{\mathrm{PS}}\right)^{2}$ at fixed values of $r_{0}^{\chi} \mu_{R}$ (b). In (b) we cannot include data at $\beta=4.2$ due to the missing value of the renormalisation factor $Z_{\mathrm{P}}$.

\begin{tabular}{|l|r|r|r|}
\hline Quantity & median & statistical & systematic \\
\hline \hline$m_{\text {up,down }}[\mathrm{MeV}]$ & 3.54 & $(19)$ & $(+16-17)$ \\
$\bar{\ell}_{3}$ & 3.50 & $(9)$ & $(+9-30)$ \\
$\bar{\ell}_{4}$ & 4.66 & $(4)$ & $(+4-33)$ \\
$f_{0}[\mathrm{MeV}]$ & 121.5 & $(0.1)$ & $(+1.1-0.1)$ \\
$r_{0}[\mathrm{fm}]$ & 0.420 & $(9)$ & $(+10-11)$ \\
$|\Sigma|^{1 / 3}[\mathrm{MeV}]$ & 270 & $(5)$ & $(+3-4)$ \\
$f_{\pi} / f_{0}$ & 1.0755 & $(6)$ & $(+8-94)$ \\
\hline
\end{tabular}

Table 1: Summary of fit results, determined from the weighted distribution of a number of $\mathscr{O}(80)$ different fits. The first error is of statistical origin while the second, the asymmetric one, accounts for the systematic uncertainties. $\Sigma$ and $m_{u, d}$ are renormalised in the $\overline{\mathrm{MS}}$ scheme at the renormalisation scale $\mu=2 \mathrm{GeV}$, as the values of $Z_{\mathrm{P}}$ are in the $\overline{\mathrm{MS}}$ scheme at scale $2 \mathrm{GeV}$. The scale is set by $f_{\pi}=130.7 \mathrm{MeV}$.

The results of these fits can be found in table 2. We give statistical and systematic errors separately, the systematic one being asymmetrical. The results are obtained by performing $\mathscr{O}(80)$ fits, which differ in fit-range, finite size correction formulae and in the order of $\chi \mathrm{PT}$. The final result is obtained as the median of the corresponding weighted distribution over all fits. The statistical error is determined using the bootstrap method with 1000 samples. The systematic uncertainty is estimated from the $68 \%$ confidence interval of the weighted distribution. For details see Ref. [8]. The fit results for the determination of physical quantities and the low energy constants listed in table 2 belong to the most precise determination of their kind world-wide.

\section{Results adding dynamical strange and charm quarks}

The very nice results for mass degenerate quarks discussed in the last section motivates to go one step beyond this setup. The ETM collaboration has by now included the strange and the charm 
degrees of freedom in their simulations and they are the first collaboration to perform such studies.

We introduce a dynamical strange quark by adding a twisted heavy mass-split doublet $\chi_{h}=$ $\left(\chi_{c}, \chi_{s}\right)$, thus also introducing a dynamical charm in our framework. As shown in [18], a real quark determinant can in this case be obtained if the mass splitting is taken to be orthogonal in isospin space to the twist direction. We thus choose the construction $[4,18]$

$$
S_{h}=a^{4} \sum_{x}\left\{\bar{\chi}_{h}(x)\left[D[U]+m_{0, h}+i \mu_{\sigma} \gamma_{5} \tau_{1}+\mu_{\delta} \tau_{3}\right] \chi_{h}(x)\right\}
$$

where $m_{0, h}$ is the untwisted bare quark mass for the heavy doublet, $\mu_{\sigma}$ the bare twisted mass - the twist is this time along the $\tau_{1}$ direction - and $\mu_{\delta}$ the mass splitting along the $\tau_{3}$ direction.

The bare mass parameters $\mu_{\sigma}$ and $\mu_{\delta}$ of the non-degenerate heavy doublet are related to the physical renormalised strange and charm quark masses via [4]

$$
\begin{aligned}
& \left(m_{s}\right)_{\mathrm{R}}=Z_{\mathrm{P}}^{-1}\left(\mu_{\sigma}-Z_{\mathrm{P}} / Z_{\mathrm{S}} \mu_{\delta}\right), \\
& \left(m_{c}\right)_{\mathrm{R}}=Z_{\mathrm{P}}^{-1}\left(\mu_{\sigma}+Z_{\mathrm{P}} / Z_{\mathrm{S}} \mu_{\delta}\right),
\end{aligned}
$$

where $Z_{\mathrm{P}}$ and $Z_{\mathrm{S}}$ are the renormalisation constants of the pseudoscalar and scalar quark densities, respectively, computed in the massless standard Wilson theory. In order to tune to maximal twist, we will use a rather economical, while accurate method $[9,18,19]$, where the choice $a m_{0, l}=$ $a m_{0, h} \equiv 1 / 2 \kappa-4$ is made.

Tuning to maximal twist, i.e. $\kappa=\kappa_{c r i t}$, is then achieved in the same way as for the $N_{f}=2$ case discussed above by demanding that the PCAC mass vanishes at $\kappa_{\text {crit }}$. For the quenched [25] and, as seen above, the $N_{\mathrm{f}}=2$ case [8], this method has been found to be successful in providing the expected $\mathscr{O}(a)$ improvement and effectively reducing residual $\mathscr{O}\left(a^{2}\right)$ discretisation effects in the region of small quark masses [24].

\subsection{Results: $f_{\mathrm{PS}}, m_{\mathrm{PS}}$ and Chiral Fits}

We concentrate in this section on the analysis of the simplest and phenomenologically relevant observables involving up and down valence quarks. These are the light charged pseudoscalar decay constant $f_{\mathrm{PS}}$ and the light charged pseudoscalar mass $m_{\mathrm{PS}}$.

The present simulations with dynamical strange and charm quarks, sitting at, or varying around, their nature given masses, should allow for a good measure of the impact of strange and charm dynamics on the low energy sector of QCD and the electroweak matrix elements. As a first step, one can determine the low energy constants of chiral perturbation theory $(\chi \mathrm{PT})$. In contrast to standard Wilson fermions, an exact lattice Ward identity for maximally twisted mass fermions allows for extracting the charged pseudoscalar decay constant $f_{\mathrm{PS}}$ from the relation

$$
f_{\mathrm{PS}}=\frac{2 \mu_{l}}{m_{\mathrm{PS}}^{2}}\left|\left\langle 0\left|P_{l}^{1}(0)\right| \pi\right\rangle\right|
$$

without need to specify any renormalisation factor, since $Z_{\mathrm{P}}=1 / Z_{\mu}$ [3]. We have performed fits to NLO SU(2) continuum $\chi \mathrm{PT}$ at $\beta=1.95$ and $\beta=1.90$, separately and combined.

We thus simultaneously fit our data for the pseudoscalar mass and decay constant to the following formulae, where the contributions $F, D$ and $T$ parametrise finite size corrections, discretisation 
effects and NNLO $\chi$ PT effects, respectively, below:

$$
\begin{aligned}
m_{\mathrm{PS}}^{2}(L) & =\chi_{\mu}\left(1+\xi l_{3}+D_{m_{\mathrm{PS}}^{2}} a^{2}+\xi^{2} T_{m_{\mathrm{PS}}^{2}}\right) F_{m_{\mathrm{PS}}^{2}} \\
f_{\mathrm{PS}}(L) & =f_{0}\left(1-2 \xi l_{4}+D_{f_{\mathrm{PS}}} a^{2}+\xi^{2} T_{f_{\mathrm{PS}}}\right) F_{f_{\mathrm{PS}}},
\end{aligned}
$$

with the pseudoscalar mass squared at tree level defined as $\chi_{\mu} \equiv 2 B_{0} \mu_{l}$ and the chiral expansion parameter by $\xi \equiv \chi_{\mu} /\left(4 \pi f_{0}\right)^{2}$.

Systematic errors can arise from several sources: finite volume effects, neglecting of higher orders in $\chi \mathrm{PT}$ and finite lattice spacing effects. These different corrections are accounted for explicitly in eq. (3.4). Finite volume corrections are described by the rescaling factors denoted by $F_{m_{\mathrm{PS}}^{2}}$ and $F_{f_{\mathrm{PS}}}$, computed in the continuum theory. Notice that the discretisation effects present in the neutral pion mass, can generate peculiar finite volume corrections which have been recently analysed in ref. [23]. Here we use, however, the resummed expressions derived by Colangelo, Dürr and Haefeli (CDH) in [15] which describe the finite volume effects in our simulations very well.

Because of the automatic $\mathscr{O}(a)$ improvement of the twisted mass action at maximal twist, the leading order discretisation artefacts in the chiral formulae of (3.4) are at least of $\mathscr{O}\left(a^{2}\right)$, and $\mathscr{O}\left(a^{2} \mu\right)$ for $m_{\mathrm{PS}}^{2}$. The regime of quark masses and lattice spacings at which we have performed the simulations is such that $\mu_{l} \gtrsim a \Lambda_{\mathrm{QCD}}^{2}$. In the associated power counting, at maximal twist, the NLO $\operatorname{tm} \chi$ PT expressions for the charged pion mass and decay constant preserve their continuum form.

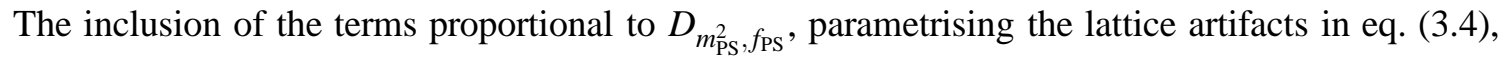
represents an effective way of including sub-leading discretisation effects appearing at NNLO.

To set the scale at each lattice spacing, we determine $a \mu_{\text {phys }}$, the value of $a \mu_{l}$ at which the ratio $\sqrt{m_{\mathrm{PS}}^{2}(L=\infty)} / f_{\mathrm{PS}}(L=\infty)$ assumes its physical value. We can then use the value of $f_{\mathrm{PS}}$, or equivalently $m_{\mathrm{PS}}$, to calculate the lattice spacing $a$ in fm from the corresponding physical value. We also perform a chiral fit combining two different lattice spacings. With only two different values of $\beta$, that are in fact fairly close to each other, a proper continuum limit analysis cannot be performed. Instead, we treat this combined fit as a check on the presence of lattice artefacts and the overall consistency of the data.

In order to estimate the statistical errors affecting our fitted parameters, we generate at each of the $\mu_{l}$ values 1000 bootstrap samples for $m_{\mathrm{PS}}$ and $f_{\mathrm{PS}}$ extracted from the bare correlators, organised by blocks. For each sample, and combining all masses, we fit $m_{\mathrm{PS}}^{2}$ and $f_{\mathrm{PS}}$ simultaneously as a function of $\mu_{l}$. The parameter set from each of these fits is then a separate bootstrap sample for the purposes of determining the error on our fit results. By resampling $f_{\mathrm{PS}}$ and $m_{\mathrm{PS}}$ on a perconfiguration basis, correlations between these quantities are taken into account.

We show our final fits in fig. 3 and summarize the results in table 2. The predictions for $\bar{l}_{3}$ and $\bar{l}_{4}$ are in good agreement and with our two-flavour predictions [8] and with other recent lattice determinations $[22,21]$.

An interesting question in the situation with a dynamical strange and charm degree of freedom is the tuning of the mass parameters $\mu_{\sigma}$ and $\mu_{\delta}$ in the heavy doublet of the action in eq. (3.1). In this work, we fix the values of $\mu_{\sigma}$ and $\mu_{\delta}$ by requiring that the simulated kaon mass $m_{K}$ and $D$ meson mass $m_{D}$ approximately take their physical values. Fig. 4 shows the quality of such a tuning. For the rather involved extraction of the kaon mass $m_{K}$ and the D-meson mass $m_{D}$ we refer to ref. [27]. As can be seen, at $\beta=1.95$ we indeed succeeded to tune the mass parameters correctly 


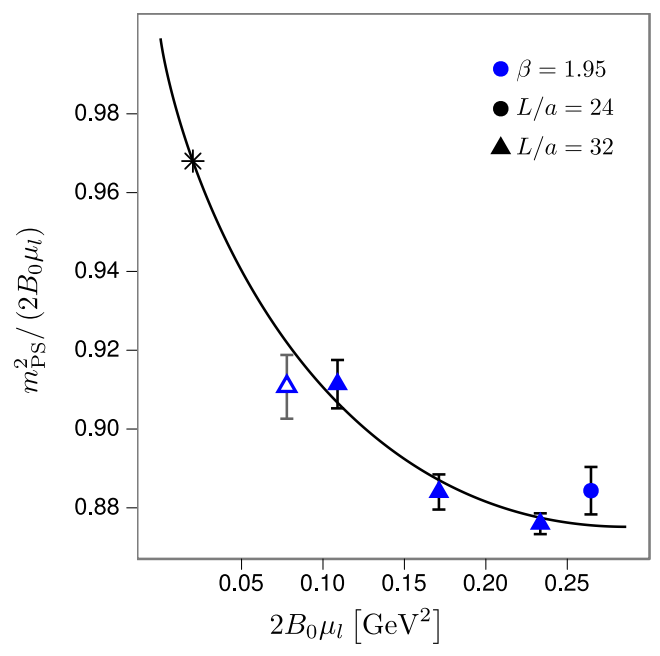

(a)

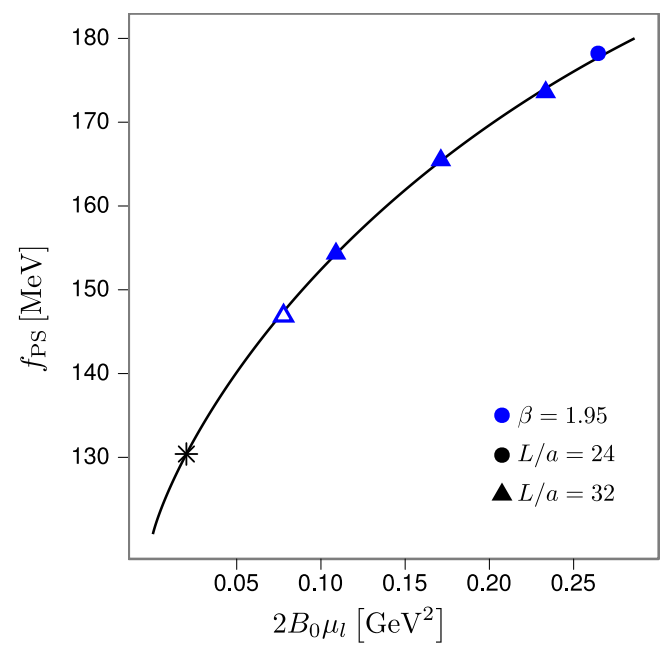

(b)

Figure 3: (a) The charged pseudoscalar mass ratio $m_{\mathrm{PS}}^{2} /\left(2 B_{0} \mu_{l}\right)$ and (b) the pseudoscalar decay constant $f_{\mathrm{PS}}$ as a function of $2 B_{0} \mu_{l}$ fitted to SU(2) chiral perturbation theory. The scale is set by the value of $2 B_{0} \mu_{l}$ at which the ratio $f_{\mathrm{PS}}^{[L=\infty]} / m_{\mathrm{PS}}^{[L=\infty]}$ assumes its physical value [16] (black star). The lattice gauge coupling is $\beta=1.95$ and the twisted light quark mass ranges from $a \mu_{l}=0.0025$ to 0.0085 corresponding to a range of the pseudoscalar mass $270 \lesssim m_{\mathrm{PS}} \lesssim$ $490 \mathrm{MeV}$. The kaon and $D$ meson masses are tuned to their physical value. The lightest point (open symbol) has not been included in the chiral fit.

\begin{tabular}{lr}
\hline \hline & $\beta=1.95$ \\
\hline \hline$\overline{l_{3}}$ & $3.70(7)(26)$ \\
$\bar{l}_{4}$ & $4.67(3)(10)$ \\
$f_{0}[\mathrm{MeV}]$ & $121.14(8)(19)$ \\
$\left\langle r^{2}\right\rangle_{s}^{\mathrm{NLO}}\left[\mathrm{fm}^{2}\right]$ & $0.724(5)(23)$ \\
\hline$a(\beta=1.95)[\mathrm{fm}]$ & $0.0782(6)$ \\
\hline
\end{tabular}

Table 2: Results of the fits to SU(2) $\chi \mathrm{PT}$ for the ensemble at $\beta=1$.95. Predicted quantities are: the low energy constants $\bar{l}_{3,4}$, the charged pseudoscalar decay constant in the chiral limit $f_{0}$ and the pion scalar radius $\left\langle r^{2}\right\rangle_{s}^{N L O}$. The first quoted error is from the chiral fit at $\beta=1.95$, the second error is the systematic uncertainty.

while at $\beta=1.9$ we are missing the physical value of the kaon mass which necessitates a retuning. We have performed such a retuning successfully as demonstrated by the corresponding data point in fig. 4(a).

\section{Conclusion}

In this contribution we have discussed a particular formulation of lattice QCD, maximally twisted mass fermions. We have shown that with this $\mathscr{O}(a)$-improved action precise results in the light meson and the strange baryon sector can be obtained. In the course of this investigation, it has been demonstrated that twisted mass fermions at maximal twisted indeed scale with $a^{2}$ towards the continuum limit and that even these remaining $a^{2}$ corrections are very small giving rise to a well 


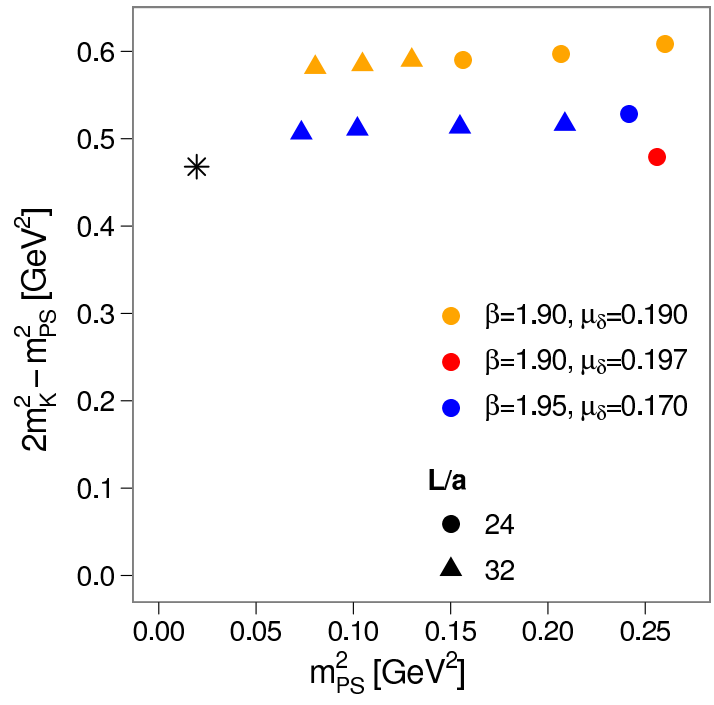

(a)

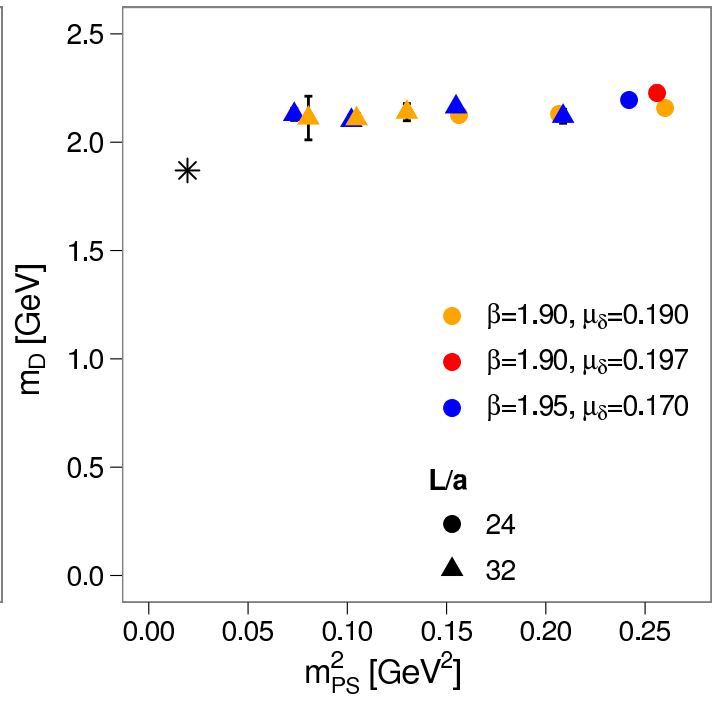

(b)

Figure 4: (a): $2 m_{K}^{2}-m_{\mathrm{PS}}^{2}$, and (b): $m_{D}$, as a function of $m_{\mathrm{PS}}^{2}$, for $\beta=1.95$ (blue) and $\beta=1.90$ (orange). The physical point is shown (black star). The kaon and $D$ meson masses appear to be properly tuned at $\beta=1.95$. The ensembles at $\beta=1.90, \mu_{\delta}=0.190$ have a larger value of the strange quark mass, while the red point at $\beta=1.90, a \mu_{\delta}=0.197$ appears to be well tuned. Data points have been scaled with the lattice spacing $a=0.08585(53) \mathrm{fm}$ for $\beta=1.90$, and $a=0.07820(59) \mathrm{fm}$ for $\beta=1.95$, obtained in this work and where the errors are only statistical.

controlled continuum limit extrapolation of lattice results. Fitting the data for $f_{\pi}$ and $m_{\pi}$ it became possible to extract a number of physical quantities and low energy constants of chiral perturbation theory very precisely, see table 2 .

Encouraged by these results, we have described first simulations for the situation when a dynamical strange and charm quark are included. We have demonstrated that it is possible to tune to physical $K$ - and $D$-meson masses. In addition the good agreement of the $N_{f}=2$ and $N_{f}=2+1+1$ simulations, visible when comparing the values for the low energy constants $f_{0}, \bar{l}_{3}$ and $\bar{l}_{4}$ with those of table 2 indicates that also for the latter case lattice artefacts, at least in the light meson sector, are small. This offers the promising possibility to obtain precise physical results also with dynamical up, down, strange and charm simulations for the first time.

\section{Acknowledgments}

We thank all other members of the ETM Collaboration for a very fruitful collaboration and many valuable discussions. The HPC resources for this project have been made available by the computer centres of Barcelona, Groningen, Jülich, Lyon, Munich, Paris and Rome (apeNEXT), which we thank for enabling us to perform this work. This work has also been supported in part by the DFG Sonderforschungsbereich/Transregio SFB/TR9-03, and by GENCI (IDRIS - CINE S), Grant 2009-052271.

\section{References}

[1] C. Alexandrou et al., arXiv:0910.2419 [hep-lat]. 
[2] K. G. Wilson, Phys. Rev. D10, 2445 (1974).

[3] ALPHA Collaboration, R. Frezzotti, P. A. Grassi, S. Sint and P. Weisz, JHEP 08, 058 (2001), hep-lat/0101001.

[4] R. Frezzotti and G. C. Rossi, JHEP 10, 070 (2004), hep-lat / 0407002.

[5] ETM Collaboration, P. Boucaud et al., Phys. Lett. B650, 304 (2007), arXiv : hep-lat / 0701012.

[6] ETM Collaboration, B. Blossier et al., JHEP 04, 020 (2008), arXiv: 0709.4574 [hep-lat] .

[7] ETM Collaboration, P. Boucaud et al., Comput. Phys. Commun. 179, 695 (2008), arXiv:0803.0224 [hep-lat].

[8] ETM Collaboration, R. Baron et al, arXiv:0911.5061 [hep-lat].

[9] R. Frezzotti and G. C. Rossi, JHEP 08, 007 (2004), hep-lat / 0306014.

[10] ETM Collaboration, P. Dimopoulos et al., arXiv:0908.0451 [hep-lat] .

[11] R. Sommer, Nucl. Phys. B411, 839 (1994), hep-lat/9310022.

[12] S. Weinberg, Physica A96, 327 (1979).

[13] J. Gasser and H. Leutwyler, Ann. Phys. 158, 142 (1984).

[14] J. Gasser and H. Leutwyler, Nucl. Phys. B250, 465 (1985).

[15] G. Colangelo, S. Dürr and C. Haefeli, Nucl. Phys. B721, 136 (2005), hep-lat / 0503014.

[16] Particle Data Group Collaboration, C. Amsler et al., Phys. Lett. B667, 1 (2008).

[17] ETM Collaboration, R. Baron et al., arXiv:0911.5244 [hep-lat].

[18] R. Frezzotti and G. C. Rossi, Nucl. Phys. Proc. Suppl. 128 (2004) 193

[19] T. Chiarappa et al., Eur. Phys. J. C 50 (2007) 373

[20] ETM Collaboration, R. Baron et al., PoS LAT2008 (2008) 094

[21] S. Necco, PoS CONFINEMENT8, 024 (2008), arXiv:0901.4257 [hep-lat].

[22] E. E. Scholz, arXiv:0911.2191 [hep-lat].

[23] G. Colangelo, U. Wenger and J. M. S. Wu, arXiv:1003.0847 [hep-lat] .

[24] R. Frezzotti, G. Martinelli, M. Papinutto and G. C. Rossi, JHEP 04, 038 (2006), hep-lat/0503034.

[25] K. Jansen, M. Papinutto, A. Shindler, C. Urbach and I. Wetzorke, Phys. Lett. B619, 184 (2005), hep-lat/0503031.

[26] R. Baron et al., arXiv:1004.5284 [hep-lat].

[27] R. Baron et al., arXiv:1005.2042 [hep-lat].

[28] S. Dürr et al., Science 322 (2008) 1224, arXiv:0906.3599 [hep-lat]. 